\title{
Multi-Objective Weighted Clustering Algorithm Minimizing Jointly the Costs of Mission and Communication in Wireless Sensor Network
}

\author{
Hicham Ouchitachen \\ LMACS, Sultan Moulay \\ Slimane University \\ B.P. 523, Faculty of Sciences \\ and Technology \\ Beni Mellal, Morocco
}

\author{
Abdellatif Hair \\ LMACS, Sultan Moulay \\ Slimane University \\ B.P. 523, Faculty of Sciences \\ and Technology \\ Beni Mellal, Morocco
}

\author{
Najlae Idrissi \\ TIAD, Sultan Moulay Slimane \\ University \\ B.P. 523, Faculty of Sciences \\ and Technology \\ Beni Mellal, Morocco
}

\begin{abstract}
Wireless sensor networks (WSNs) have recently gained the attention of researchers in many challenging aspects. The energy conservation is one of the most important issues in these networks. Due to the limited access to the nodes, both the network structure and the manner of communication between the nodes decide the energy expenditure in WSNs. One of the best solutions, in this context, is to cluster the network. This paper presents a new clustering algorithm for solving the energetic constraint in WSNs. More precisely, a critical network is considered, where each sensor satisfies its own missions depending on its locations. In addition to fulfill their mission, the sensor tries to maintain a good neighboring nodes quality. First, the mission and communication costs of sensors are minimized jointly using Sensor's Genetic Algorithm (SGA), then the Multi-Objective Weighted Clustering Algorithm (MOWCA) is developed. It aims at dividing a network into different clusters and at selecting the best performing sensors in terms of power to communicate with the Base Station (BS). MOWCA is based on tree critical parameters. $\mathrm{DD}_{\mathrm{i}}$ : Degree Difference of sensor $\mathrm{i}, \mathrm{DC}_{\mathrm{i}}$ : Sum of distances between sensor $\mathrm{i}$ and its neighbors and $\mathrm{DM}_{\mathrm{i}}$ : Mission distance of sensor i. Later on and in order to balance energy consumed in different formed clusters, the Base Station Genetic Algorithm (BGA) is established. Simulation results demonstrate that the proposed algorithms are very advantageous in terms of convergence to the appropriate locations and are so efficient in regards to energy conservation in WSNs.
\end{abstract}

\section{Keywords}

Wireless Sensor Networks, Energy Conservation, Mission Satisfaction, Communication Quality, Optimal Placement.

\section{INTRODUCTION}

In recent years, wireless communication and sensor technologies have seen tremendous growth. Wireless Sensors Networks (WSNs) have emerged as a promising research domain and have been used in a wide variety of applications [1]. They have been used in health field [2,3], Environmental field [4,5,6], and smart home-field [7]. By means of this recent technology, it becomes possible to interact with the surrounding environment through the use of multiple tiny sensors. WSNs use sensors to co-operatively monitor complex environmental or physical conditions. Such sensors are generally equipped with communication capabilities and data processing to collect data and to route information back to a Base Station (BS) [8]. WSNs are examples of resourceconstrained networks in which the processing resources, the storage and the energy are limited [9]. This constraint of energy is a critical issue that needs to be tackled so that WSNs can be widely employed. In WSN, the power source consists of a battery with a limited energy budget which results in a finite lifetime of nodes. In addition, it could be impossible or inconvenient to recharge the battery because nodes may be deployed in a hostile or unpractical environment [10]. In the last few years, several studies have established for the extension of nodes' battery life as much as possible. A survey that offers a comprehensive view of energy-saving solutions in WSNs while taking applications' requirements into consideration is presented in [11].

It is very important to note that in WSN both the network structure and the manner of communication between the nodes decide the energy expenditure. On the plain network, hierarchical structures are generally preferred due to their reliability and improved energy conservation. Clustering is the prominent hierarchical architecture. Cluster formation is one of the early proposed methods for energy efficient operation in WSNs [12].

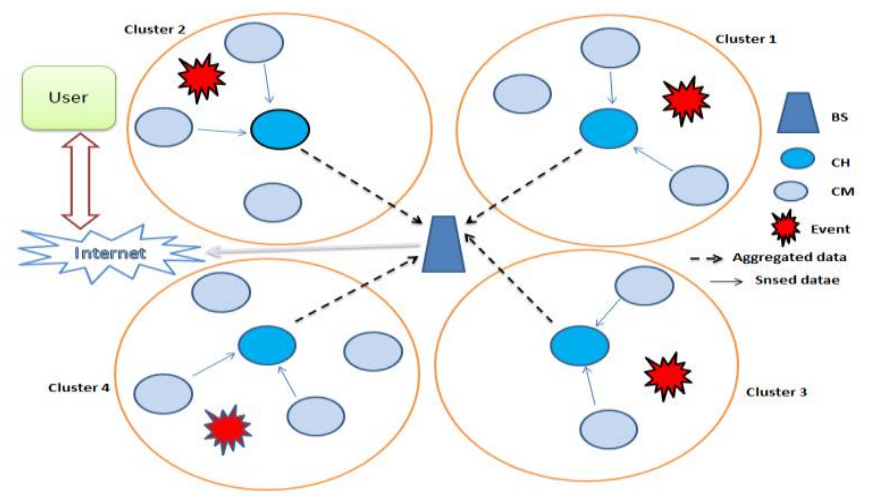

Fig 1: Clustering in WSN

In clustering, the sensor nodes are divided into different virtual groups according to a set of rules [13]. Some nodes are selected as Cluster Heads (CHs) and the other nodes are called Cluster Members (CMs) [14]. CHs are responsible for managing the $\mathrm{CMs}$, receiving and processing data from them. Only CHs communicate with the BS directly, while each CM simply communicates with its own CH (See Fig 1). Usually, $\mathrm{CHs}$ consume more energy than their $\mathrm{CMs}$, since $\mathrm{CHs}$ have the responsibility of network organization, data gathering, and long distance data transmissions with the BS [15]. Clustering the nodes in WSNs is performed with different objectives and 
purposes presented in [16]. The energy conservation is the most important and common goal of all these objectives.

\subsection{Objectives}

Motivated by the importance of network structure and the manner of communication between the nodes in the energy expenditure under WSN, this work considers jointly those factors (Network structure and communication manner). More precisely, the main objective is to develop a clustering algorithm for solving the energetic constraint in WSNs by the joint minimization of mission and communication cost. In other words, the proposed algorithm aims at ensuring both efficient satisfaction of sensors' mission and improving the quality of communication between them while minimizing jointly the costs of these two operations.

\subsection{Structure}

The paper is organized as follows. Section 2 deals with related works. The details of proposed schemes are presented in Section 3. Subsection 3.1 recalls the interest of SGA algorithm in terms of joint minimization of mission and communication costs. Different phases of MOWAC algorithm are detailed in subsection 3.2. Subsection 3.3 shows how to achieve the optimal position of BS using our algorithm BGA, in order to balancing energy consumed in formed clusters. The numerical result, the possible comparisons, the various analyzes and the performances of proposed algorithms are provided in Section 4. Finally, Section 5 concludes our paper.

\section{RELATED WORK}

Many works have been considered for tacking clustering issue and finding good location of nodes in WSNs. For the first challenge, in the last decade, a lot of approaches have been proposed for finding an energy efficient solution for one of the following clustering problems : Cluster size [17], transmission power load balancing between cluster members[18, 19], and $\mathrm{CH}$ selection [ 20, 21].

Numerous clustering algorithms for WSNs have been proposed in [22] typically aiming at reducing the power consumption. Another algorithm based on a clever strategy of cluster head $(\mathrm{CH})$ selection, residual energy of the $\mathrm{CHs}$ and the intra-cluster distance for cluster formation is presented in [23].

In 2014, one of the most important surveys on WSN clustering algorithms has been presented in [16] where the authors describe some important clustering approaches in WSNs. Some other hierarchical clustering protocols including LEACH, HEED, TEEN, APTEEN, and EECS are discussed in [24]. In [25] LEACH and its recent advances are studied. A neural network based clustering approaches are presented in [26] which focuses on five neural network based algorithms: ART, ART1, FUZZY ART, IVEBF, and EBCS. In [27], the transmission load assignment in WSNs is modeled as a game. This work focuses on a cluster-based and surveillanceoriented sensor network.

Another challenge, in this context, is to find a good location for the BS based on initial topological information such as distances between sensor nodes and the BS. However, such schemes are not resource aware and may not lead to the best placement for the BS. In general the sink placement problem is NP-complete [28] and finding the best position of sink is very hard. In recent years, several papers report on BS positioning $[29,30,31,32]$ and mainly design the network to ensure energy conservation and network lifetime extension. Since the optimal location of BS is one of the important factors in the present approach, recent attempts made in this research area are reminded. In 2015, some new protocols are presented in $[33,34,35]$. Others approaches are discussed in $[36,37,38,39]$.

However, none of these papers considers jointly the cost of mission and the quality of communication, also the network considered is not critical. The work we consider in this paper is clearly different in several critical aspects. We propose a network with multiple nodes with controllable mobility in two-dimensional space. This network focuses on providing a good communication quality or getting letter quality services, except that the nodes of the sensor networks are additionally interested in the satisfaction of their missions. The works established in these critical cases are so rare. Our previous papers [40] and [41] are two recent proposals in this context, where we proposed some new algorithms that accurately find the best locations of sensors and BS while minimizing the average energy consumed in the network.

\section{PROPOSED SCHEMES}

\subsection{Network Model}

We suppose that a set of $\mathrm{n}$ sensors is deployed in a geographic area of interest to supervise a given physical phenomenon. The topology of a WSNs is represented by the graph $\mathrm{G}=(\mathrm{C}$, $\mathrm{E})$, where $\mathrm{C}=\{1,2 \ldots \mathrm{n}\}$ is a set of $\mathrm{n}$ sensors and $\mathrm{E} \subset \mathrm{C} \times \mathrm{C}$ is the set of wireless links between the various sensors. $\mathrm{C}_{\mathrm{v}(\mathrm{i})}$ is the neighbor set of the sensor i. In Table 1, we present the meanings of the notations used in our modeling.

\subsection{Joint Minimization of Mission and Communication Costs}

Before determining the different clusters constituting the network, we briefly recall the objective of the first phase of our approach which is the reduction of mission and communication costs of each node. For this, we used our SAG algorithm presented in [ouchi1]. SAG aims to find the optimal locations of sensors by solving the optimization problem given by (1) as follows:

$$
\begin{gathered}
\operatorname{minf}(x, y)=\sum_{i} f_{i}^{s}\left(d_{i s}\right)+\sum_{i} \sum_{j \in C_{v(i)}} c f_{i j}^{c}\left(d_{i j}\right) \\
\text { Subject of }\left(x_{i}, y_{i}\right) \in S_{i} \quad \forall(i, j) \in C \times C_{v(i)}
\end{gathered}
$$

The pseudo code of the SAG algorithm is given below.

Algorithm 1. SGA algorithm

$$
\begin{aligned}
& \text { Data: }\left(\mathrm{x}_{\mathrm{i}}^{\mathrm{m}}, \mathrm{y}_{\mathrm{i}}^{\mathrm{m}}\right),\left(\mathrm{x}_{\mathrm{i}}^{\mathrm{c}}, \mathrm{y}_{\mathrm{i}}^{\mathrm{c}}\right), \mathrm{i} \in \mathrm{C} \\
& \text { Result: }\left(\mathrm{x}_{\mathrm{i}}^{\text {op }}, \mathrm{y}_{\mathrm{i}}^{\text {op }}\right), \mathrm{i} \in \mathrm{C} \\
& \text { initialization of population } \mathrm{P} ; \\
& \text { while No convergence } \\
& \text { do } \\
& \qquad \mathrm{P}^{\prime}:=\text { Selection of parents in } \mathrm{P} ; \\
& \qquad \mathrm{P}^{\prime}:=\text { Apply the crossing operator on } \mathrm{P}^{\prime} ; \\
& \mathrm{P}^{\prime}:=\text { Apply the mutation operator on } \mathrm{P}^{\prime} ; \\
& \quad \mathrm{P}^{\prime}:=\text { Replace old parents by their descendants } \mathrm{P}^{\prime} ; \\
& \quad \text { Evaluate } \mathrm{P}^{\prime} ; \\
& \text { End }
\end{aligned}
$$


Table 1. Summary of notations

\begin{tabular}{|c|c|}
\hline Notations & Meaning \\
\hline 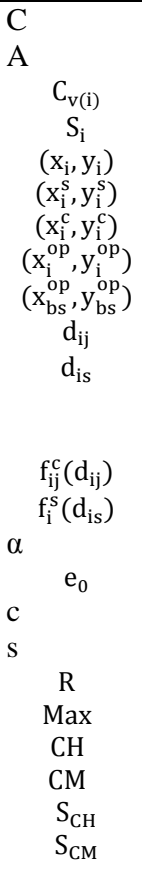 & $\begin{array}{l}\text { Set of sensors } \\
\text { Set of active sensors } \\
\text { Neighbors set of sensor i } \\
\text { Area in where each sensor i can move freely } \\
\text { Current position of sensor i } \\
\text { Mission's position of sensor i } \\
\text { Communication's position of sensor i } \\
\text { Optimal location of sensor i } \\
\text { Optimal location of base station } \\
\text { Distance between sensors i and j } \\
\text { Distance between current and mission's } \\
\text { position of a sensor } \\
\text { Cost of communication between sensors i et j } \\
\text { Mission cost of sensor i. } \\
\text { Attenuation parameter } \\
\text { Energy needed to transmit one unit of data to BS } \\
\text { Communication factor } \\
\text { Surveillance factor } \\
\text { Transmission radius of a sensor } \\
\text { Maximal number of sensors managed by cluster head } \\
\text { Cluster head } \\
\text { Cluster member } \\
\text { Cluster heads set } \\
\text { Cluster members set }\end{array}$ \\
\hline
\end{tabular}

\subsection{Description of our Algorithm MOWAC}

After the calculation of the optimal position for each sensor $i$ using SAG, this section presents the main phase of the given approach. Indeed, to contribute to solve the energetic constraint and to optimize the resources in mission-critical sensor networks, the MOWAC algorithm is developed based on the following parameters:

- $\quad \mathbf{D D}_{\mathbf{i}}$ : Degree Difference of sensor $\mathrm{i}$ that is the difference between the degree of sensor i (Number of sensors within its transmission radius $\mathrm{R}$ ) and a predefined ideal node number Max in a cluster.

- $\mathbf{D C}_{\mathbf{i}}$ : Sum of distances between sensor $\mathrm{i}$ and its neighbors.

- $\quad \mathbf{D M}_{\mathbf{i}}$ : Mission distance of sensor i.

To determine the different clusters, the MOWAC algorithm follows these steps:

- Step 1: - Determinate the neighbor set $\mathrm{C}_{\mathrm{v}(\mathrm{i})}$ of each sensor $\mathrm{i}$, where $\mathrm{C}_{\mathrm{v}(\mathrm{i})}$ is defined by:

$$
C_{\mathrm{v}(\mathrm{i})}=\left\{\mathrm{j} \in \mathrm{C} /\left[\left(\mathrm{x}_{\mathrm{i}}^{\mathrm{op}}-\mathrm{x}_{\mathrm{j}}^{\mathrm{op}}\right)^{2}+\left(\mathrm{y}_{\mathrm{i}}^{\mathrm{op}}-\mathrm{y}_{\mathrm{j}}^{\mathrm{op}}\right)^{2}\right]^{\frac{1}{2}} \leq\right.
$$$$
\text { Calculate the degree } d_{i} \text { of each sensor } i
$$

defined by: $\mathrm{d}_{\mathrm{i}}=\operatorname{Card}\left(\mathrm{C}_{\mathrm{v}(\mathrm{i})}\right)$

- Step 2: Compute the degree difference of each sensor i by this formula: $D_{i}=\left|d_{i}-\operatorname{Max}\right|$

- Step 3: Compute the sum $\mathrm{DC}_{\mathrm{i}}$ of the distances between sensor $\mathrm{i}$ and its neighbors. That is:

$$
D C_{i}=\sum_{j \in C_{v(i)}}\left[\left(x_{i}{ }^{o p}-x_{j}{ }^{o p}\right)^{2}+\left(y_{i}{ }^{o p}-y_{j}{ }^{o p}\right)^{2}\right]^{1 / 2}
$$

- Step 4: Calculate the parameter $\mathrm{DM}_{\mathrm{i}}$ which represents the distance between the optimal position $\left(\mathrm{x}_{\mathrm{i}}^{\mathrm{op}}, \mathrm{y}_{\mathrm{i}}^{\mathrm{op}}\right)$ of the sensor $i$ and the position of sensor's mission $\left(x_{i}^{s}, y_{i}^{s}\right)$ : $\mathrm{DM}_{\mathrm{i}}=\left[\left(\mathrm{x}_{\mathrm{i}}{ }^{\text {op }}-\mathrm{x}_{\mathrm{i}}{ }^{\mathrm{m}}\right)^{2}+\left(\mathrm{y}_{\mathrm{i}}{ }^{\text {op }}-\mathrm{y}_{\mathrm{i}}{ }^{\mathrm{m}}\right)^{2}\right]^{1 / 2}$

- Step 5: Calculate the combined weight $\mathrm{CW}_{\mathrm{i}}$ as follow:

$$
\mathrm{CW}_{\mathrm{i}}=w_{1} \times D D_{i}+w_{2} \times D C_{i}+w_{3} \times D M_{i}
$$

Where $w_{1}, w_{2}$ and $w_{3}$ are different weights such that $w_{1}+w_{2}+w_{3}=1$

- Step 6: Select the sensor with the minimum combined weight $\mathrm{CW}_{\mathrm{i}}$ as a cluster head.

- Step 7: Eliminate the chosen cluster head and its neighbors from the set of original sensor nodes.

- Step 8: Execute Steps 1 to 7 for the remaining sensors until each one is assigned to a cluster.

After the execution of these steps successively, the different clusters are formed and all sensor nodes are grouped into clusters with correspond $\mathrm{CHs}$.

Figure 1 shows the flowchart of our algorithm.

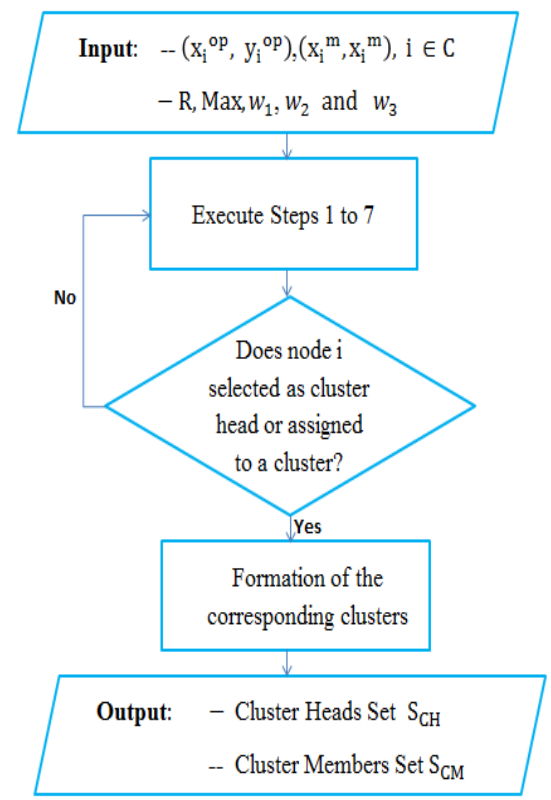

Fig 2: MOWAC flowchart

\subsubsection{Explanatory example}

This subsection provides on illustration on how the MOWAC algorithm is running by considering twelve sensors characterized by their initial factors as shown in Table 2 .

Table 2. Sensors initial factors

\begin{tabular}{|c|c|c|}
\hline Sensor $\mathbf{i}$ & $\left(\mathbf{x}_{\mathbf{i}}{ }^{\mathbf{m}}, \mathbf{\mathbf { y } _ { \mathbf { i } }}{ }^{\mathbf{m}}\right)$ & $\left(\mathbf{x}_{\mathbf{i}}{ }^{\mathbf{o p}}, \mathbf{y}_{\mathbf{i}}{ }^{\mathbf{o p}}\right)$ \\
\hline 1 & $(50,60)$ & $(50,64.3)$ \\
\hline 2 & $(80,60)$ & $(75,65)$ \\
\hline 3 & $(120,60)$ & $(122,66)$ \\
\hline 5 & $(170,60)$ & $(165,65.5)$ \\
\hline 6 & $(70,80)$ & $(65,85.5)$ \\
\hline 7 & $(140,90)$ & $(136,90.5)$ \\
\hline 8 & $(70,110)$ & $(69.8,115)$ \\
\hline
\end{tabular}




\begin{tabular}{|c|c|c|}
\hline 9 & $(150,110)$ & $(145,114)$ \\
\hline 10 & $(140,130)$ & $(133,137)$ \\
\hline 11 & $(110,150)$ & $(105,155)$ \\
\hline 12 & $(60,150)$ & $(57.4,156)$ \\
\hline
\end{tabular}

Also the parameters that are necessary for the operation of the algorithm are defined as follows:

- The threshold number Max is set at 6, which means that a cluster head can conveniently manage 6 sensors.

- $\quad$ The three weights $w_{1}, w_{2}$ and $w_{3}$ are respectively set to the values $0.4,0.3$ and 0.3 .

Our algorithm proceeds as follows:

Step 1: The neighbors set $\boldsymbol{C}_{\boldsymbol{v}(\boldsymbol{i})}$ of each sensor and its degree $\boldsymbol{d}_{\mathbf{i}}$ are obtained as shown in Table 3 .

Table 3. $N_{\mathrm{i}}$ and $d_{\mathrm{i}}$ values for each sensor $i$

\begin{tabular}{|l|c|l|}
\hline Sensor i & $\boldsymbol{N}_{\mathbf{i}}$ & $\boldsymbol{d}_{\mathbf{i}}$ \\
\hline 1 & $\{2,5,7\}$ & 3 \\
\hline 2 & $\{1,3,5,7\}$ & 4 \\
\hline 3 & $\{2,4,6,9\}$ & 4 \\
\hline 4 & $\{3,6,9\}$ & 3 \\
\hline 5 & $\{1,2,7,8\}$ & 4 \\
\hline 6 & $\{3,4,8,9,10\}$ & 5 \\
\hline 7 & $\{1,2,5,8,11,12\}$ & 6 \\
\hline 8 & $\{5,6,7,9,10,11,12\}$ & 7 \\
\hline 9 & $\{3,4,6,8,10,11\}$ & 6 \\
\hline 10 & $\{6,8,9,11\}$ & 4 \\
\hline 11 & $\{7,8,9,10,12\}$ & 5 \\
\hline 12 & $\{7,8,11\}$ & 3 \\
\hline
\end{tabular}

Step 2: The degree difference $D D_{\mathrm{i}}$ of each sensor $\mathrm{i}$ is derived using formula 1.

Step 3: The different distances $\boldsymbol{D} \boldsymbol{C}_{\boldsymbol{i}}$ are calculated by the formula 3. For example,

$$
\begin{gathered}
D C_{1}=\left(\left[(50-75)^{2}+(64.3-65)^{2}\right]^{\frac{1}{2}}+\left[(50-65)^{2}+\right.\right. \\
\left.\left.(64.3-85.5)^{2}\right]^{\frac{1}{2}}+\left[(50-69.8)^{2}+(64.3-115)^{2}\right]^{\frac{1}{2}}\right) \\
=105
\end{gathered}
$$

Step 4: For each sensor i, the distance $\boldsymbol{D} \boldsymbol{M}_{\boldsymbol{i}}$ is calculated by the formula 4. For example,

$$
\begin{aligned}
D M_{1} & =\left(\left[(50-50)^{2}+(64.3-60)^{2}\right]^{\frac{1}{2}}\right) \\
& =4.3
\end{aligned}
$$

Step 5: For each sensor i, the combined weight $\boldsymbol{C} \boldsymbol{W}_{\boldsymbol{i}}$ is calculated using formula 5 . For example: $\boldsymbol{C} \boldsymbol{W}_{\mathbf{1}}=0.4 \times 3+0.3 \times 105+0.3 \times 4.3$

After Step 5, the various parameters $\boldsymbol{D} \boldsymbol{D}_{\boldsymbol{i}}, \boldsymbol{D} \boldsymbol{M}_{\boldsymbol{i}}, \boldsymbol{D} \boldsymbol{C}_{\boldsymbol{i}}$ and $\boldsymbol{C} \boldsymbol{W}_{\boldsymbol{i}}$ are calculated and listed in Table 4.
Step 6: The sensor having the smallest value of combined weight $\boldsymbol{C} \boldsymbol{W}_{\mathbf{i}}$ is chosen as a cluster head. Table 5 shows that $\boldsymbol{C} \boldsymbol{W}_{10}$ is the minimum value of the combined weight. Thus, the sensor 10 is selected as the first cluster head. Figure 3 presents the obtained results.

Table 4. $D C_{\mathrm{i}}, D M_{\mathrm{i}} D D_{\mathrm{i}}$ and $C W_{\mathrm{i}}$ values for each sensor $i$

\begin{tabular}{|r|r|r|r|r|}
\hline Sensor i & \multicolumn{1}{|c|}{$D C_{\mathrm{i}}$} & \multicolumn{1}{c|}{$D M_{\mathrm{i}}$} & \multicolumn{1}{c|}{$D D_{\mathrm{i}}$} & \multicolumn{1}{c|}{$C W_{\mathrm{i}}$} \\
\hline 1 & 105 & 4,3 & 3 & 33,99 \\
\hline 2 & 155 & 7,0711 & 2 & 49,42133 \\
\hline 3 & 171 & 6,3246 & 2 & 53,99738 \\
\hline 4 & 133 & 7,433 & 3 & 43,3299 \\
\hline 5 & 163 & 7,2863 & 2 & 51,88589 \\
\hline 6 & 184 & 4,0311 & 1 & 56,80933 \\
\hline 7 & 269 & 5,004 & 0 & 82,2012 \\
\hline 8 & 298 & 6,7082 & 1 & 91,81246 \\
\hline 9 & 253 & 6,4031 & 0 & 77,82093 \\
\hline 10 & 100 & 9,8995 & 2 & 33,76985 \\
\hline 11 & 220 & 7,0711 & 1 & 68,52133 \\
\hline 12 & 148 & 6,5391 & 3 & 47,56173 \\
\hline
\end{tabular}

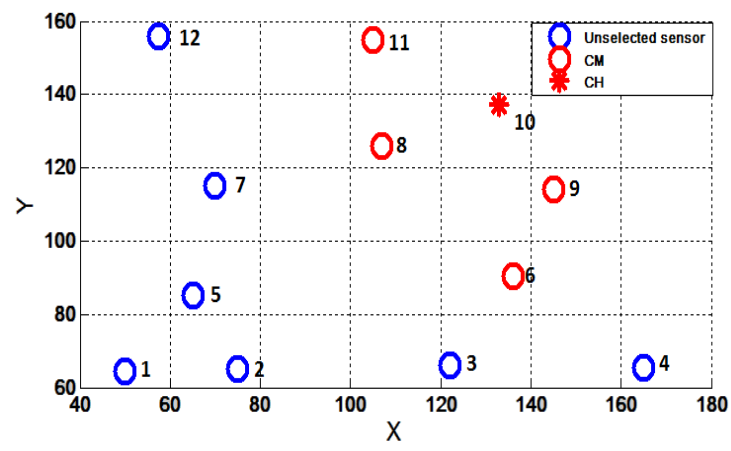

Fig 3: Select of the first cluster head

Step 7: The chosen cluster head (CH: Sensor 10) and its neighbors (CMs: Sensors 6, 8, 6 and 11 are eliminated from the set of original sensor nodes.

Figure 4 shows the results obtained after removing the first cluster head and its neighbors.

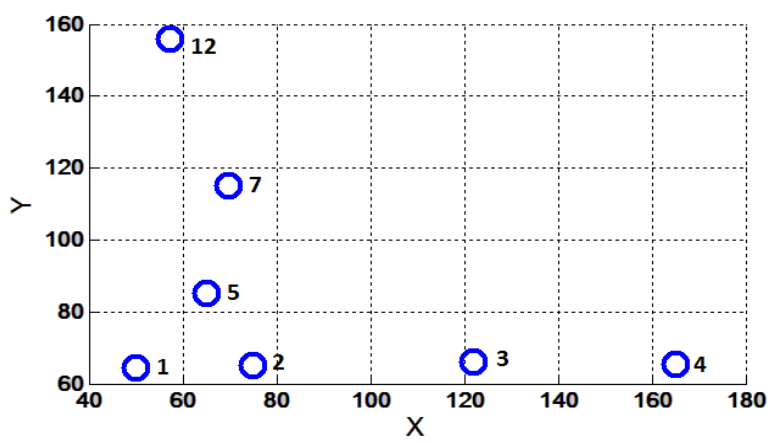

Fig 4: The remaining sensor nodes after the first iteration 
The steps from 1 to 7 are repeated for the remaining sensors until each sensor is assigned to a cluster. The final results of clustering are shown in Figure 5.

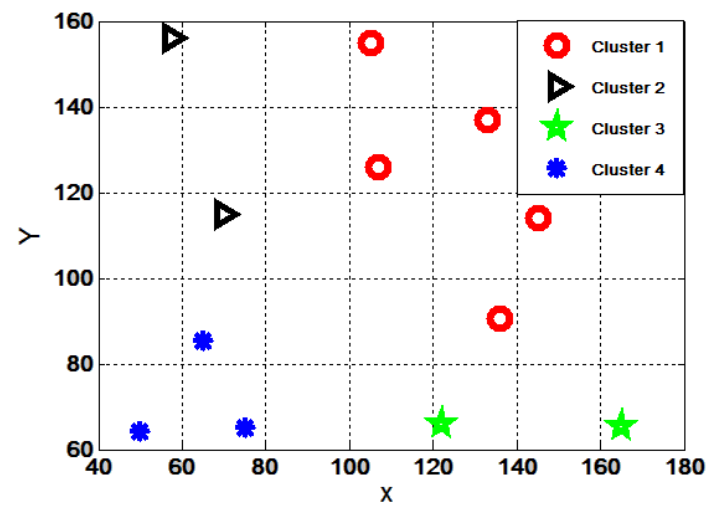

Fig 5: The final results of clustering using MOWAC

\subsection{Balancing Consumed Energy in Formed Clusters by Placing BS in the Best Location}

The main goal here is to determine the best position BS relatively to different clusters formed. For this, two algorithms namely: Simulated Annealing Algorithm (SAA) and Base station's Genetic Algorithm (BGA) [ouchi2] are used. The pseudo code of the BGA algorithm is presented below.

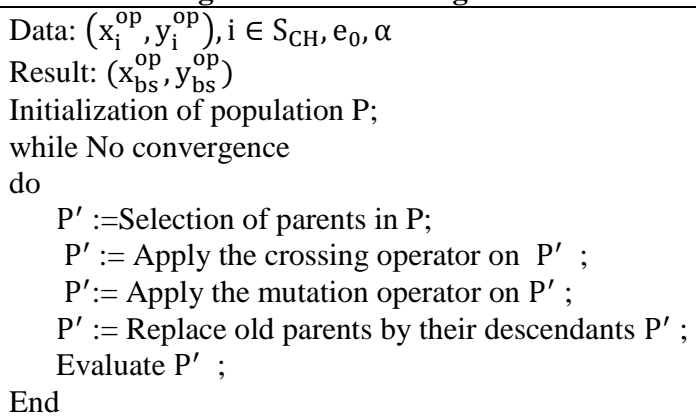

\section{SIMULATIONS AND RESULTS}

This section displays numerical results given by the three algorithms SGA, BGA and MOWAC. The cost functions and parameters are defined as follows [41]:

- $\mathrm{f}_{\mathrm{ij}}^{\mathrm{c}}\left(\mathrm{d}_{\mathrm{ij}}\right)=100 \exp \left(10 / 12\left(\log _{2}\left(10^{6}\left(\mathrm{~d}_{\mathrm{ij}}\right)\right)\right)\right.$;

- $\mathrm{f}_{\mathrm{ij}}^{\mathrm{s}}\left(\mathrm{d}_{\mathrm{is}}\right)=5 \exp \left(10^{-2} \mathrm{~d}_{\mathrm{is}}-1\right)$;

- $\mathrm{C}=\{1,2 \ldots 12\}$ and $\mathrm{e}_{0}=15 \cdot 10^{-3} \mathrm{~mJ}$;

- $w_{1}=0.4, w_{2}=0.3, w_{3}=0.3$ and $\mathrm{Max}=6$

- For $\mathrm{i} \in \mathrm{C}$, the values of $\left(\mathrm{x}_{\mathrm{i}}^{\mathrm{m}}, \mathrm{y}_{\mathrm{i}}^{\mathrm{m}}\right)$ and $\left(\mathrm{x}_{\mathrm{i}}{ }^{\mathrm{op}}, \mathrm{y}_{\mathrm{i}}{ }^{\mathrm{op}}\right)$ are shown in Table 2.

The considered network is shown in Fig.6, which consists of 12 nodes, where PM and PI denote respectively the mission and communication's position of each sensor i.

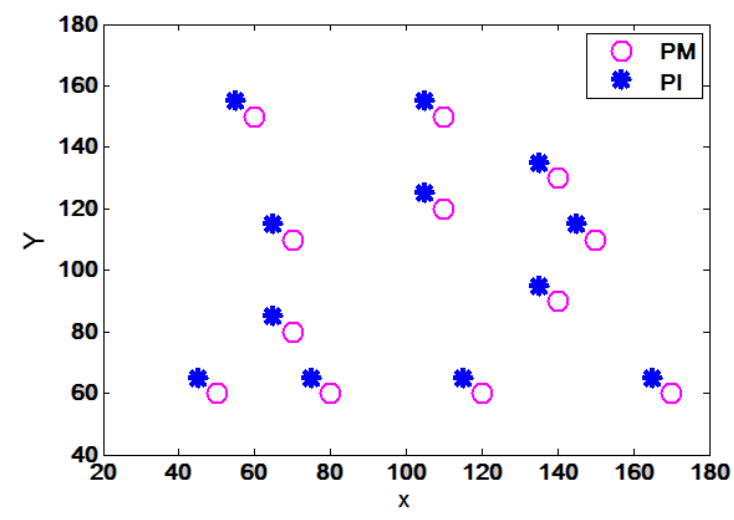

Fig 6: The mission and communication's position of each sensor i

The best locations of sensors calculated using SGA are represented in Figure 7.

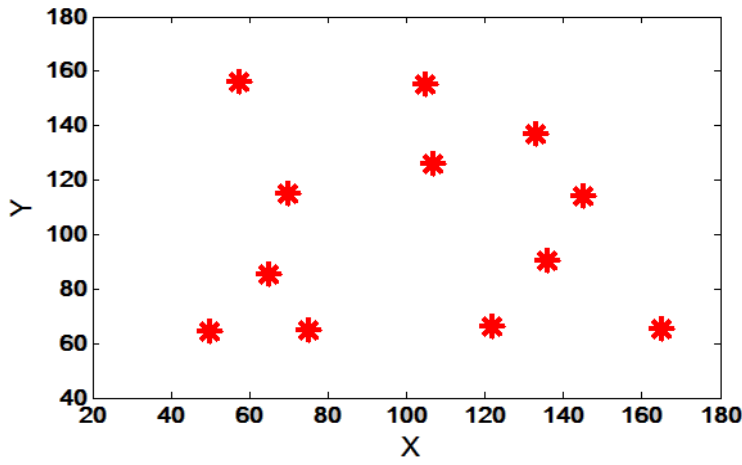

Fig 7: The best locations of sensors given by SGA

After balancing mission and communication costs for each sensor, the MOWAC algorithm is executed to form the different clusters and determine explicitly the two sets $S_{C H}$ and $S_{C M}$. Later on, the base station is placed in its best position relatively to the different clusters formed. The results of this clustering are shown in Figure 8, where the four clusters formed are distinguished by colours as follows: $1 \mathrm{~s}$ cluster (Blue), $2^{\text {nd }}$ cluster (Green), $3^{\text {th }}$ cluster (Purple) and $4^{\text {th }}$ cluster (Pink).

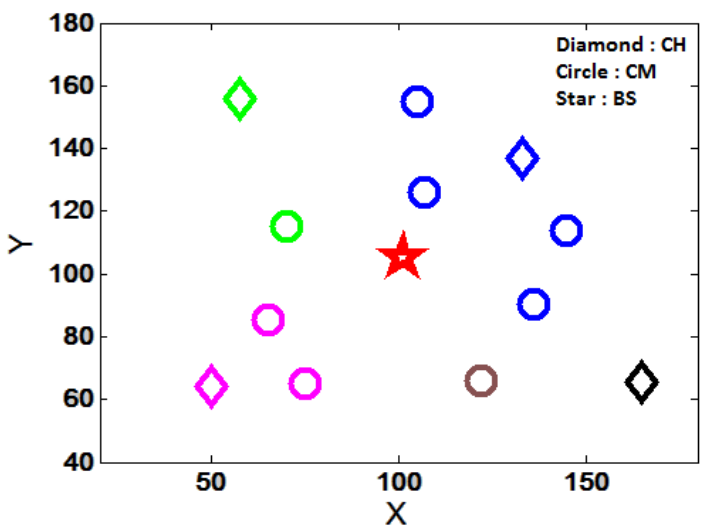

Fig 8: Different clusters given by MOWAC

Note that the best position of BS is calculated using two methods (SAA and BGA). The total energy consumed by active sensors in the network is computed. Figure 9 illustrates this energy in both cases. 


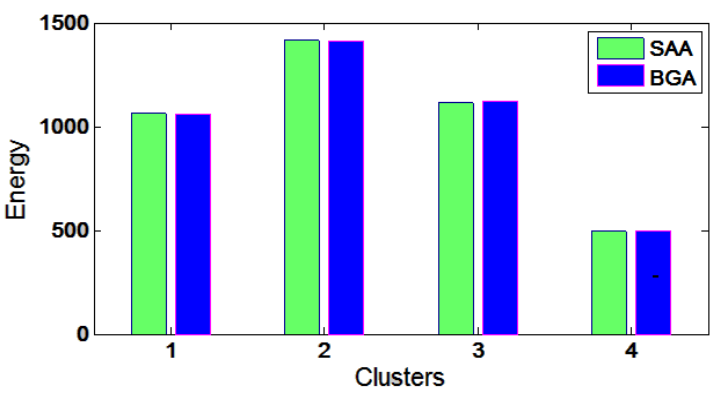

Fig 9: Consumed energy in different clusters

The comparison between SAA and BGA shows that the amount of consumed energy is the same in both cases. While the BGA algorithm is very advantageous in terms of convergence, this fact is shown clearly in Figure 10.

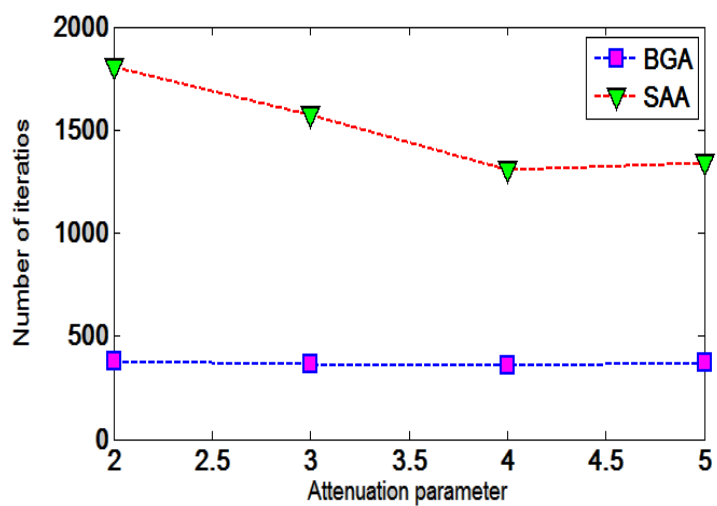

Fig 10: The convergence of SAA and BGA

The performance of the BGA e BGA algorithm against SAA is justified by the advanced techniques of genetic algorithms that have passed from the stage of basic research to applied research. Indeed, in terms of convergence, SAA is negatively influenced by the choice of the initial solution which is one of the most important criteria for SAA. So, to achieve the final solution, SAA searches only in the vicinity of the initial solution. By cons, after coding the chromosomes, the BGA algorithm (See Algorithm 2) can start with any initial population, then performs a global search to reach the best solution. Thus, BGA evolves this population by selecting the best individuals. Then, thanks to the operation of croissant, it evolves also these individuals with possible mutations.

Later on, another distinguishing point of BGA algorithm is noted. Indeed, for optimizing the objective function; BGA does not impose any regularity (Continuity, differentiability, convexity, ...) about this function.

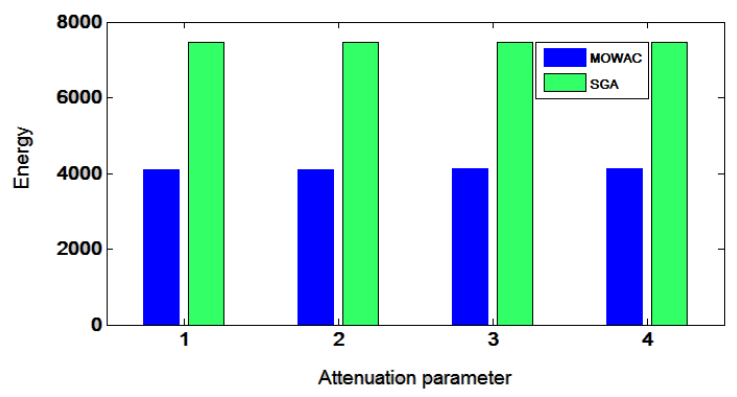

Fig 11: If necessary, the images can
By comparing the MOWAC algorithm with SAG, it seems obvious, from Figure 11, that MOWAC is more efficient. Specifically, it is clear that the total consumed energy in the network has decreased remarkably. This means that MOWAC saves a lot of energy which is to date a great challenge for researchers in the area of WSN.

On one hand, the MOWAC performance is justified by the fact of introducing the different metrics $D D_{\mathrm{i}}, D C_{\mathrm{i}}$ and $D M_{\mathrm{i}}$ (for each sensor i) in the function to be optimized. Indeed, the MOWAC algorithm benefited greatly from the importance of multi-objective optimization used. This technique allows MOWAC to consider the different critical parameters in the network studied namely, the mission cost, the communication cost and also the distance between sensors and BS. On the other hand, thanks to the clustering performed by MOWAC, only the best performing sensors in terms of power are selected to communicate with BS, which is very advantageous as regards energy consumption in WSN.

\section{CONCLUSION}

This paper presents some new algorithms aiming at solving the energy constraint in critical WSNs where each node tries to minimize the weighted sum of mission and communication cost in a distributed way. The proposal approach is based on advanced techniques of genetic algorithms. The obtained results show that, comparing to other techniques, the presented algorithms in this work are very advantageous in terms of convergence to the optimal solution. The different simulations displays that total consumed energy in the network has decreased remarkably. This means that the presented algorithms save a lot of energy which is, up to now, a great challenge for researchers in the WSNs area.

We structure our thoughts as follows: The first phase concerns the proposition of new protocols for responding to the concern degree of mobility and self-configuration. As a second objective, we want propose a routing algorithm that incorporates the concept of cluster management. Finally, we also plan to focus on the effective management of resources in mobile sensor networks.

\section{REFERENCES}

[1] Chun Lo, Jerome P. Lynch, Mingyan Liu, Distributed model-based nonlinear sensor fault diagnosis in wireless sensor networks Mechanical Systems and Signal Processing, Volumes 66-67, January 2016, pp. 470-484.

[2] Heal, Sofia Maria Dima, Christos Panagiotou, Dimitris Tsitsipis, Christos Antonopoulos, John Gialelis, Stavros Koubias, Performance evaluation of a WSN system for distributed event detection using fuzzy logic, Ad Hoc Networks, Volume 23, December 2014, pp .87. 108.

[3] Hackmann G, Guo W, Yan G, et al. Cyber-physical codesign of distributed structural health monitoring with wireless sensor network [J]. Parallel and distributed Systems, IEEE Transaction on, 2015, 25(1), pp. 63-72.

[4] Environement : Manuel Delamo, Santiago Felici-Castell, Juan J. Pérez-Solano, Andrew Foster, Designing an open source maintenance-free Environmental Monitoring Application for Wireless Sensor Networks, Journal of Systems and Software, Volume 103, May 2015, pp. 238247 
[5] Environement F.J. Mesas-Carrascosa, D. Verdú Santano, J.E. Meroño, M. Sánchez de la Orden, A. García-Ferrer, Open source hardware to monitor environmental parameters in precision agriculture Biosystems Engineering, Volume 137, September 2015, Pages 73-83.

[6] Murat Dener, Yunus Özkök, Cevat Bostancioğlu, Fire Detection Systems in Wireless Sensor Networks, Procedia - Social and Behavioral Sciences, Volume 195, 3 July 2015, pp.1846-1850.

[7] Wang J C, Lin C H, Siahaan E, et al. Mixed sound event verification on wireless sensor network for home automation [J].Industrial Informatics, IEEE Transaction on, 2014, 10(1), pp. $802-812$

[8] Alessandro Di Stefano, Aurelio La Corte, Marco Leotta, Pietro Lió, Marialisa Scatá, It measures like me: An IoTs algorithm in WSNs based on heuristics behavior and clustering methods, Ad Hoc Networks, Volume 11, Issue 8, November 2013, pp. 2637-2647.

[9] Atslands R. Rocha, Luci Pirmez, Flávia C. Delicato, Érico Lemos, Igor Santos, Danielo G. Gomes, José Neuman de Souza, WSNs clustering based on semantic neighborhood relationships, Computer Networks, Volume 56, Issue 5, 30 March 2012, pp.1627-1645.

[10] Muruganantham Arunraja, Veluchamy Malathi, Erulappan Sakthivel, Energy conservation in WSN through multilevel data reduction scheme Microprocessors and Microsystems, Volume 39, Issue 6, August 2015, pp. 348-357.

[11] Rault T, Bouabdallah A, Challal Y. Energy-efficiency in wireless sensor networks: a top-down review approach. Comput Netw 2014;67: pp. 22-104.

[12] Tarek AlSkaif, Manel Guerrero Zapata, Boris Bellalta, Game theory for energy efficiency in Wireless Sensor Networks: Latest trends, Journal of Network and Computer Applications, Volume 54, August 2015, pp. 33-61.

[13] L.Malathi, R.K. Gnanamurthy, Krishnan Chandrasekaran, Energy efficient data collection through hybrid unequal clustering for wireless sensor , Computers \& Electrical Engineering, Available online 22 July 2015.

[14] Zhou X, Wu M, Xu J. BPEC: an energy-aware distributed clustering algorithm in WSNs. J Comput Res Develop 2009;46(5): pp. 723-30.

[15] Jiang Zhu, Chung-Horng Lung, Vineet Srivastava, A hybrid clustering technique using quantitative and qualitative data for wireless sensor networks, Ad Hoc Networks, Volume 25, Part A, February 2015, pp. 38-53.

[16] M. Mehdi Afsar, Mohammad-H. Tayarani-N, Clustering in sensor networks: A literature survey, Journal of Network and Computer Applications, Volume 46, November 2014, pp. 198-226.

[17] Yang Y, Lai C, Wang L, Wang X. An energy-aware clustering algorithm via game theory for wireless sensor network s. In: 2012 12th International conference on control, automation and systems (ICCAS), Jeju Island, Korea (South); 2012.

[18] Lin X-H, Kwok Y-K, Wang H, Xie N. A game theoretic approach to balancing energy consumption in heterogeneous wireless sensor networks. Wirel Commun Mob Comput 2015; 15(1): pp. 170-91.

[19] Lee D, Shin H, Lee C. Game theory-based resource allocation strategy for clustering based wireless sensor network. In: Proceedings of the sixth international conference on ubiquitous information management and communication. Kuala Lumpur, Malaysia: ACM; 2012, pp. 112.

[20] Zeng Y, Chen Z, Qiao C, Xu L. A cluster header election scheme based on auction mechanism for intrusion detection in MANET. In: 2011 International conference on network computing and information security (NCIS), vol. 2. Guilin, China: IEEE; 201, pp. $433-7$.

[21] Tan L, Zhang S, Qi J. Cooperative cluster head selection based on cost sharing game for energy-efficient wireless sensor networks. J Comput Inf Syst 2012;8 (9), pp. 3623-33.

[22] Pengfei Zhang, Gaoxi Xiao, Hwee-Pink Tan, Clustering algorithms for maximizing the lifetime of wireless sensor networks with energy-harvesting sensors, Computer Networks, Volume 57, Issue 14, 4 October 2013, pp. 2689-2704.

[23] Tarachand Amgoth, Prasanta K. Jana, Energy-aware routing algorithm for wireless sensor networks, Computers \& Electrical Engineering, Volume 41, January 2015, pp. 357-367.

[24] Joshi A, Lakshmi Priya M. A survey of hierarchical routing protocols in wireless sensor network. MES J Technol Manag 2011, pp. 67-71.

[25] Jindal P, Gupta V. Study of energy efficient routing protocols of wireless sensor networks and their further researches: a survey. J Comput Sci Commun Eng 2013.

[26] Subhai C, Malarkan S, Vaithinathan K. A survey on energy effi cient neural network based clustering models in wireless sensor networks . In: International conference on emerging trends in VL SI, embedded system, nano electronics and telecommunication system (ICEVENT), Tamil Nadu, India; 2013, pp. 1-6.

[27] Lin X-H, Kwok Y-K, Wang H, Xie N. A game theoretic approach to balancing energy consumption in heterogeneous wireless sensor networks. Wirel Commun Mob Comput 2015;15(1), pp.170-91.

[28] A. Bogdanov, E. Maneva, S. Riesenfeld, Power-Aware Base Station positioning for Sensor Networks, in: Proceedings of the 23rd AnnualJoint Conference of the IEEE Computer and Communications Societies (INFOCOM 2004), Hong Kong, March 2004.

[29] K. Akkaya, M. Younis, M. Bangad, Sink repositioning for enhanced performance in wireless sensor networks, Computer Networks 49 (2005), pp. 512-534

[30] Z. Vincze, R. Vida, A. Vidacs, Deploying multiple sinks in multi-hop wireless sensor networks, in: Pervasive Services, IEEE International Conference, 2007, pp. 5563.

[31] E.I. Oyman, C. Ersoy, Multiple sink network design problem in large scale wireless sensor networks, in: Communications, 2004 IEEE International Conference , 2004, pp. 3663-3667. 
[32] A. Bogdanov, E. Maneva, S. Riesenfeld, Power-Aware Base Station positioning for Sensor Networks, in: Proceedings of the 23rd AnnualJoint Conference of the IEEE Computer and Communications Societies( INFOCOM 2004), Hong Kong, March 2004.

[33] Demin Gao, Haifeng Lin, Xiaofeng Liu, Routing protocol for k-anycast communication in rechargeable wireless sensor networks, Computer Standards \& Interfaces, Volume 43, January 2016, pp. 12-20.

[34] Saeid Mottaghi, Mohammad Reza Zahabi, Optimizing LEACH clustering algorithm with mobile sink and rendezvous nodes, AEU - International Journal of Electronics and Communications, Volume 69, Issue 2, February 2015, pp. 507-514

[35] E.I. Oyman, C. Ersoy, Multiple sink network design problem in large scale wireless sensor networks, in: Communications, 2004 IEEE International Conference, 2004, pp. 3663-3667.

[36] A. Bogdanov, E. Maneva, S. Riesenfeld, Power-aware base station positioning for sensor networks, in: INFOCOM 2004. Twenty Third AnnualJoint Conference of the IEEE Computer and Communications Societies, 2004, pp. 585.
[37] Liu W, Lu K, Wang J, Huang L, Wu DO. On the throughput capacity of wireless sensor networks with mobile relays. IEEE Trans Veh Technol 2012;61(May (4)), pp. 1801-9.

[38] Konstantopoulos C, Pantziou G, Gavalas D, Mpitziopoulos A, Mamalis B. A rendezvous-based approach enabling energy-efficient sensory data collection with mobile sinks. IEEE Trans Parallel Distrib Syst 2012;23(May (5)), pp. 809-17.

[39] Liang W, Luo J, Xu X. Prolonging network lifetime via a controlled mobile sink in wireless sensor networks. In: Global telecommunications conference (IEEE GLOBECOM 2010). 2010, December, pp. 1-6.

[40] Ouchitachen H, Hair A, Idrissi, N. Joint mission and communication aware node placement problem in mission-specific mobile sensor networks. In: Codes, Cryptography and Communication Systems (WCCCS). 27-28 November, 2014 (Indexed in IEEE Xplore).

[41] Ouchitachen H, Hair A, Idrissi, N. Optimal placement of sensors in mission-specific mobile sensor networks. In: TELKOMNIKA Indonesian Journal of Electrical Engineering Vol. 15, No. 3, September 2015, pp. 401408 . 